\title{
Coupling finite elements and proper generalized decompositions
}

\author{
A. Ammar ${ }^{1}$, F. Chinesta ${ }^{2}$, E. Cueto ${ }^{3}$ \\ ${ }^{1}$ Laboratoire de Rhéologie, UMR CNRS-UJF-INPG \\ 13 rue de la Piscine, BP 53 Domaine Universitaire \\ F-38041 Grenoble cedex 9, France \\ Amine.Ammar@ujf-grenoble.fr \\ ${ }^{2}$ EADS Corporate Foundation International Chair \\ GeM: UMR CNRS-Ecole Centrale de Nantes \\ 1 rue de la Noe, BP 92101, F-44321 Nantes cedex 3, France \\ Francisco.Chinesta@ec-nantes.fr \\ ${ }^{3}$ Aragón Institute of Engineering Research \\ University of Zaragoza \\ Maria de Luna s/n, E-50018 Zaragoza, Spain \\ ecueto@unizar.es
}

\begin{abstract}
Numerous models encountered in science and engineering remain nowadays, despite the impressive recent progresses attained in computational simulation techniques, intractable when the usual and well experienced discretization techniques are applied for their numerical simulation. Thus, different challenging issues are waiting for the proposal of new alternative advanced simulation techniques. Separated representations offer the possibility to address some challenging models with CPU time savings of some orders of magnitude. In other cases, they allowed to address models never until now solved. The number of published works concerning this kind of approximation remains quite reduced, and then, numerous difficulties that were successfully circumvented in the context of more experienced discretization techniques, as is the case of the finite element method, must be considered again within the separated representation framework. One of these issues in the one that concerns the treatment of localized behavior of model solutions. This work focuses on this topic and propose an efficient finite element (or extended finite element) enrichment of usual separated representation.
\end{abstract}


keyword Separated representations; Finite sums decomposition; Finite Elements; Enriched Finite Elements; Proper Generalized Decomposition

\section{Introduction}

Numerous models encountered in science and engineering remain nowadays, despite the impressive recent progresses attained in computational simulation techniques, intractable when the usual and well experienced discretization techniques are applied for their numerical simulation. Thus, different challenging issues are waiting for the proposal of new alternative advanced simulation techniques, the brut force approach being no more a valuable alternative.

A first challenging issue concerns the treatment of highly multidimensional models arising from quantum mechanics or kinetic theory descriptions of solids and complex fluids, including micro and nano-structured materials [9]. Other multidimensional models are encountered in biology, genetics and chemistry where the so called chemical master equation plays a key role [18]. Curse of dimensionality also arises in stochastic models involving numerous random variables [16]. The main challenge in the treatment of this kind of models is related to their multidimensionality because when one applies standard mesh based discretization techniques the number of degrees of freedom involved scales exponentially with the dimension of the space concerned. Thus, in high dimensions, usual meshes cannot be defined at all, needing the proposal of new advanced strategies able to circumvent the terrible curse of dimensionality. Until now, the treatment of this kind of models was restricted to the ones defined in moderate multidimensional spaces where for example the sparse grid based methods works [6].

We proposed in some of our former works [1] [2] a separated representation strategy able to circumvent the curse of dimensionality that those models involve. Thus, given a model involving the unknown field $u\left(\mathbf{x}_{1}, \mathbf{x}_{2}, \cdots, \mathbf{x}_{D}\right)$, with $\left(\mathbf{x}_{1}, \mathbf{x}_{2}, \cdots, \mathbf{x}_{D}\right) \in \Omega_{1} \times \Omega_{2} \times \cdots \times \Omega_{D}$, where $\Omega_{i} \subset \mathbb{R}^{d_{i}}, \forall i, 1 \leq i \leq D$, the solution is searched as

$$
u\left(\mathbf{x}_{1}, \mathbf{x}_{2}, \cdots, \mathbf{x}_{D}\right) \approx \sum_{i=1}^{i=N} \alpha_{i} F_{1}^{i}\left(\mathbf{x}_{1}\right) \cdots F_{D}^{i}\left(\mathbf{x}_{D}\right)
$$

where the dimension of the model is $D I M=\sum_{j=1}^{j=D} d_{j}$.

Obviously, if a mesh is defined in each domain $\Omega_{i}$ consisting of $N_{n}^{i}$ nodes, the whole mesh of $\Omega=\Omega_{1} \times \cdots \times \Omega_{D}$ involves $\prod_{i=1}^{i=D} N_{n}^{i}$ nodes. We can notice that the number of degrees of freedom increases exponentially with the dimension of the space. On the contrary, by assuming the separated representation (1) the number of degrees of freedom reduces to: $N \times\left(\sum_{i=1}^{i=D} N_{n}^{i}\right)$ proving that the complexity scales linearly with the dimension of the space.

Another issue concerns the solution of transient multiscale models (usually strongly non linear and coupled, and always of high size). These models arise 
in computational mechanics (involving a large variety of constitutive behaviors, couplings etc.). In this context, the use of standard incremental discretization techniques becomes inefficient from the computational time viewpoint. Moreover, in general, these models involve different characteristic times differing of several decades. Again, alternative advanced computational techniques are needed. Pierre Ladeveze proposed several years ago a powerful technique for addressing this kind of challenging models that he called the LATIN method [13].

The LATIN method integrates many ingredients leading to a robust, powerful, efficient and accurate discretization technique especially well adapted for treating transient multi-scale non-linear models. The two most outstanding ingredients are (i) the decoupling between a linear-global problem and a nonlinear-local one, both defined in the whole space-time domain; and (ii) a spacetime separated representation of the model variables in order to accelerate the solution of the linear-global problem. The former separated representation was called by Ladeveze in the 80's "radial approximation", and in our knowledge it was the first time that separated representations were applied in computational mechanics. The interested reader can refers to [14] and the references therein for a recent overview and the state of the art of the LATIN approach in multi-scale modeling.

Thus, the radial approximation proposed by Ladeveze can be viewed as a particular case of Eq. (1) with only two coordinates, i.e. $u\left(\mathbf{x}_{1}, \mathbf{x}_{2}\right), \mathbf{x}_{1}$ representing the physical space, i.e. $\mathbf{x}_{1} \equiv \mathbf{x} \in \Omega \subset \mathbb{R}^{d}(d=2$ or $d=3)$; and $\mathbf{x}_{2}$ the time, i.e. $\mathbf{x}_{2} \equiv t \in \mathcal{I} \subset \mathbb{R}^{+}$. However, because the radial approximation was proposed before the separated representation given by Eq. (1) (originally proposed in [1] [2]) we could also state that Eq. (1) is no more than a generalization of the so called radial approximation for models involving many coordinates.

Separated representations were applied before its use in computational mechanics in other branches of the computational physics. A remarkable example concerns quantum chemistry that focuses in the modeling of atomic structure and molecular bonds. In that case, one must solve the Schrödinger equation, or some more advanced alternatives, as the fully relativistic Dirac equation. The solution of these equations is of capital interest to describe the structure and mechanics of materials at the finest scale, descriptions that can be directly applied in the framework of nanosciences and nanotechnologies, but that also allow deriving coarse grained models by applying appropriate multiscale bridges. Thus, quantum chemistry leads to "ab initio" simulations that at its turn leads to molecular dynamics simulations. Coarse graining results first in Brownian dynamics simulations and then on statistical mechanics descriptions. The coarsest scale concerns obviously the macroscopic modeling. This multiscale modeling needs accurate models and solvers at each scale as well as appropriate bridges for moving from one scale to the neighbor ones.

However, the solution of the quantum scale models is quite delicate because the wavefunction that describe for example the electronic distribution, is defined in a highly multidimensional space whose dimension scales with the number of particles that the system involves. Thus, the only possibility of representing a 
function in such a space was the use of separated representations that constitutes the basis of the Hartree-Fock and the post-Hartree-Fock approaches [7]. Despite the nice dream that this multiscale modeling (ranging from the quantum to the macroscopic scale) opened, this procedure is in fact inapplicable at present, and probably for many many years, because the starting point fails: the Schrödinger or the Dirac equations remain intractable due to the Pauli's exclusion principle constraint. Simplified models are then mandatory (see [7] for an excellent overview).

Numerous topics concerning the use of separated representation were and are being addressed in our researches. Some recent developments concern:

- From the mathematical point of view some results concerning the convergence were obtained, but nothing is known about the rate of convergence [4]. Its connection with the POD (Proper Orthogonal Decomposition) was also analyzed in [14]. In fact, introducing the separated representation into the weak formulation of the model the different functions involved in the separated representation can be computed. However, these functions that are optimal from the point of view of the strategy considered, are no more orthogonal. For this reason, we called the resulting approximation Proper Generalized Decomposition (PGD).

- From the point of view of the applications, there is a huge catalogue [15] [1] [2] [3] [8] among many others.

- From the point of view of the computing time savings, these are often of some orders of magnitude and in some cases this technique allowed solving models never until now solved, implying hundreds of dimensions, in the context of deterministic or stochastic models [2].

- We proved recently that in some non-linear-local models arising from the natural decoupling of a non-linear-global model (the LATIN strategy being the most natural choice) can be globalized by applying a separated representation. Even for strongly non-linear behaviours (e.g. thermal models involving strongly non-linear curing kinetics usually encountered in polymer or composites forming processes) the CPU time savings are impressive.

- Some proposals concerning the adaptivity and the error estimation have been done. However, further developments are needed to improve their efficiency.

- The stabilization of models involving advective terms is another issue that needs further developments. Another open issue concerns the stabilizations of mixed formulations for verifying the stability (LBB) conditions.

- Separated representations of the 3D physical space can be performed, and easily coupled with standard or enriched finite element descriptions for capturing evolving discontinuities, singularities or to perform subdomains coupling. 
This paper focuses in one of the just referred topics, the one concerning the separated representation of variables defined in usual physical spaces $(2 \mathrm{D}$ or $3 \mathrm{D})$. In [10] we applied such one approach to perform high resolution homogenization where the representative volume (a 3D cube in that case) was described using thousand nodes along each of the coordinate directions. In that case, instead of using a mesh involving $10^{9}$ degrees of freedom, we considered a separated representations that only involved few thousands of degrees of freedom. At present other groups are considering this separated representation to perform direct numerical simulation in flows involving turbulence, identification of permeability in porous media and homogenization in heterogenous media.

Recently, we extended the use of separated representations to domains others that cubes or hyper-cubes where the issue of non-homogeneous boundary conditions were also addressed [12]. However, models can also involve interfaces where the field or some of its derivatives could be discontinuous. In this case the use of separated representation seems to be delicate. We prove in this work that a coupling between the separated representation for describing the smooth solution behavior and a finite element (or an enriched finite element) description in some patches of the domain where the solution is expected to exhibit localization or some kind of discontinuity, seems to be an appealing choice to address these models.

Other issue concerns the coupling of different subdomains, each one involving a different separated representation of the solution. Thus, the required continuity conditions at those interfaces must be enforced. The present work analyzes this difficulty and propose an efficient strategy for coupling subdomains also based on the use of appropriate finite element patches. A direct consequence of this last procedure is the coupling of two subdomains, one of them involving a separated representation of the unknown fields, whereas the other one considers a finite element description. Thus, one could easily couple the finite element technology, well experienced and very powerful, with a reduced modeling performed by using separated representations in the regions of the domain where the solution is expected evolving smoothly (in many cases these regions represent the largest part of the domain in which the model is defined, as in the case of many models in fracture mechanics where the evolving crack is very localized and only affects a small region of the material domain; models in fluid-structure interaction, where that interaction only occurs in a very localized region of a larger domain, ...)

Next section revisits the algorithm related to the PGD of the unknown field. In section 3 this strategy is generalized by considering a finite element based enrichment of the separated representation for capturing localized behavior. Finally, section 4 describes some potential applications of this enriched formulation, in particular a model that exhibits localization and another one involving subdomains coupling. 


\section{The Proper Generalized Decomposition revis- ited}

For the sake of simplicity we are considering a simple $2 D$ model in wich the unknown field depends on the coordinates $x$ and $y$, defined in the domains $\Omega_{x}$ and $\Omega_{y}$ respectively. Now, the solution is searched in the form:

$$
u(x, y) \approx \sum_{i=1}^{i=N} \alpha^{i} F^{i}(x) \cdot G^{i}(y)
$$

In order to build-up this separated representation, an iterative algorithm is proposed, solving at each iteration a projection and an enrichment problem.

If we assume that the first $n$ functions in the sum has been already computed

$$
u(x, y) \approx \sum_{i=1}^{i=n} \alpha^{i} F^{i}(x) \cdot G^{i}(y)
$$

the projection stage consists of finding the $n$ best alpha-coefficients. Then, the enrichment stage lies in finding the best functions $R(x)$ and $S(y)$ such that the updated representation given by

$$
u(x, y) \approx \sum_{i=1}^{i=n} \alpha^{i} F^{i}(x) \cdot G^{i}(y)+R(x) \cdot S(y)
$$

satisfies the weak formulation for particular test functions.

We are assuming a generic equation

$$
\mathcal{K}(u(x, y))+\mathcal{L}(u(x, y))=0
$$

where $\mathcal{K}$ and $\mathcal{L}$ are two differential operators. For the sake of simplicity we are assuming that the first one only involves derivatives with respect to the $x$-coordinate, the second one involving the derivatives with respect to the other coordinate.

The computer implementation of these steps needs for a discrete representation of all the functions: $F^{i}(x), G^{i}(y), R(x)$ and $S(y)$ using a finite element interpolation in their associated domains $\Omega_{x}$ and $\Omega_{y}$. For this purpose we introduce the vectors $\mathbf{M}$ and $\mathbf{N}$ containing the shape functions associated with the meshes of $\Omega_{x}$ and $\Omega_{y}$ respectively. Finally $\mathbf{F}^{i}, \mathbf{G}^{i}, \mathbf{R}$ and $\mathbf{S}$ represent the nodal description of the associated functions. We define the following matrices related to the weak formulation of equation (5):

$$
\left\{\begin{array}{l}
\mathbb{M}=\int_{\Omega_{x}} \mathbf{M M}^{T} d \Omega_{x} \\
\mathbb{N}=\int_{\Omega_{y}} \mathbf{N N}^{T} d \Omega_{y} \\
\mathbb{K}=\int_{\Omega_{x}} \mathbf{M} \mathcal{K} \mathbf{M}^{T} d \Omega_{x} \\
\mathbb{L}=\int_{\Omega_{y}} \mathbf{N} \mathcal{L} \mathbf{N}^{T} d \Omega_{y}
\end{array}\right.
$$


These integrals take into account the specific character of each operator. For example integration by parts is used in second order operators, upwinding for stabilizing advective terms, etc.

\section{Projection stage}

In this stage we consider the test functions given by

$$
u^{*}(x, y)=\sum_{i=1}^{n} \alpha^{i *} F^{i}(x) \cdot G^{i}(y)
$$

which writes in the discrete form:

$$
u^{*}(x, y)=\sum_{i=1}^{n} \alpha^{i *} \mathbf{F}^{i T} \mathbf{M G}^{i T} \mathbf{N}
$$

Using the discrete form of function $u(x, y)$ :

$$
u(x, y)=\sum_{i=1}^{n} \alpha^{i} \mathbf{M}^{T} \mathbf{F}^{i} \mathbf{N}^{T} \mathbf{G}^{i}
$$

the weak formulation of Eq. (5) leads after numerical integration to:

$$
\sum_{i=1}^{n} \sum_{j=1}^{n} \alpha^{i *} H_{i j} \alpha^{j}=0
$$

where

$$
H_{i j}=\mathbf{F}^{i T} \mathbb{K} \mathbf{F}^{j} \cdot \mathbf{G}^{i T} \mathbb{N} \mathbf{G}^{j}+\mathbf{F}^{i T} \mathbb{M} \mathbf{F}^{j} \cdot \mathbf{G}^{i T} \mathbb{L} \mathbf{G}^{j}
$$

This system must be solved taking into account boundary and/or normality conditions. For example, we could consider a first term in the sums decomposition verifying the boundary conditions, and then the other ones must vanish on the domain boundary (this condition is enforced in the enrichment stage). In this case we must enforce $\alpha^{1}=1$.

\section{Enrichment stage}

In this stage we consider the unknown field given by

$$
u(x, y)=\sum_{i=1}^{n} \alpha^{i} F^{i}(x) \cdot G^{i}(y)+R(x) \cdot S(y)
$$

and the test functions by

$$
u^{*}(x, y)=R^{*}(x) \cdot S(y)+R(x) \cdot S^{*}(y)
$$


whose discrete form writes

$$
u^{*}(x, y)=\left[\begin{array}{ll}
\mathbf{R}^{* T} & \mathbf{S}^{* T}
\end{array}\right]\left(\begin{array}{c}
\mathbf{M}\left(\mathbf{S}^{T} \mathbf{N}\right) \\
\left(\mathbf{R}^{T} \mathbf{M}\right) \mathbf{N}
\end{array}\right)
$$

The weak form of Eq. (5) becomes, in the framework of a fixed point strategy, in:

- For a given $S(y)$ (that implies $u^{*}(x, y)=R^{*}(x) \cdot S(y)$ )

$$
\begin{aligned}
& \left(\mathbb{K} \mathbf{S}^{T} \mathbb{N} \mathbf{S}+\mathbb{M} \mathbf{S}^{T} \mathbb{L} \mathbf{S}\right) \mathbf{R}= \\
& =-\sum_{i=1}^{n} \alpha^{i}\left(\mathbb{K} \mathbf{F}^{i} \mathbf{S}^{T} \mathbb{N} \mathbf{G}^{i}+\mathbb{M} \mathbf{F}^{i} \mathbf{S}^{T} \mathbb{L} \mathbf{G}^{i}\right)
\end{aligned}
$$

- For a given $R(x)$ (that implies $u^{*}(x, y)=R(x) \cdot S^{*}(y)$ )

$$
\begin{aligned}
& \left(\mathbf{R}^{T} \mathbb{K} \mathbf{R} \mathbb{N}+\mathbf{R}^{T} \mathbb{M} \mathbf{R} \mathbb{L}\right) \mathbf{S}= \\
& =-\sum_{i=1}^{n} \alpha^{i}\left(\mathbf{R}^{T} \mathbb{K} \mathbf{F}^{i} \mathbb{N} \mathbf{G}^{i}+\mathbf{R}^{T} \mathbb{M} \mathbf{F}^{i} \mathbb{L} \mathbf{G}^{i}\right)
\end{aligned}
$$

It must be noticed that the number of the degrees of freedom involved in such non linear solution is the sum of the degrees of freedom involved in each direction (instead the product of them that mesh based discretizations strategies would have involved).

Finally, after convergence, the new approximation functions $\mathbf{F}^{n+1}$ and $\mathbf{G}^{n+1}$ are obtained by normalizing $\mathbf{R}$ and $\mathbf{S}$ :

$$
\left\{\begin{array}{l}
\mathbf{F}^{n+1}=\mathbf{R}\left(\mathbf{R}^{T} \mathbb{M} \mathbf{R}\right)^{-\frac{1}{2}} \\
\mathbf{G}^{n+1}=\mathbf{S}\left(\mathbf{S}^{T} \mathbb{N} \mathbf{S}\right)^{-\frac{1}{2}}
\end{array}\right.
$$

The projection and enrichment stages must continue until reaching convergence.

\section{Finite elements based separated representa- tions enrichment}

In this section we are modifying the technique just presented in order to capture localized behaviors such as high gradients or weak and strong discontinuities.

This kind of behaviors induces too many functional products within a standard separated representation. However, it is well known that they can be easily taken into account within the finite element framework, by using adaptive local mesh refinement in the first case or any of the techniques able to represent weak 
and strong discontinuities (meshes compatible with the interfaces or enriched approximations when the meshes are not compatible with the interfaces).

Thus, the mail goal is how combining separated representations, able to approximate the solution in the most part of the domain where the model is defined, reducing significantly the computational cost, and a localized finite element description, that even if it is expensive in nature, it only applies in a small region of the whole domain where the solution is expected to exhibit localized behavior. To this end, we propose a multi-scale approach that somewhat resembles the $s$-version of the finite element method by J. Fish [11] or the multi-scale FEM proposed by Rank [17], but in this case in a global (Ritz) and separated basis function setting.

We come back to the model described in the previous section (Eq. (5)) defined in $\Omega=\Omega_{x} \times \Omega_{y}$ and we imagine that the solution $u(x, y)$ is smooth enough everywhere except in a small region $\Omega_{l} \subset \Omega$. Now, we could imagine an approximation combining both, the separated representation and a finite element approximation, where the last one only applies in $\Omega_{l}$. We assume a mesh on $\Omega_{l}$ composed of $N_{l}$ nodes. We also assume that the finite element shape functions related to the finite element approximation cannot be expressed from the tensor product of the one dimensional bases employed to build-up the separated representation according to the description given in the previous section.

Now, the approximation in the whole domain can be written as:

$$
u(x, y)=u^{S R}(x, y)+u^{F E}(x, y)
$$

where $u^{S R}(x, y)$ is defined in the whole domain $\Omega=\Omega_{x} \times \Omega_{y}$ whereas the finite element enrichment $u^{F E}(x, y)$ is only defined within $\Omega_{l}$ vanishing in $\Omega-\Omega_{l}$. In order to ensure the continuity of the resulting approximation we must enforce the nullity of the enrichment $u^{F E}(x, y)$ on the boundary of $\Omega_{l}, \partial \Omega_{l}$.

The resulting approximation writes:

$$
u(x, y) \approx \sum_{i=1}^{i=N} \alpha^{i} F^{i}(x) \cdot G^{i}(y)+\sum_{j=1}^{j=N_{l}} \Psi_{j}(x, y) u_{j}
$$

where $\Psi_{j}(x, y)$ are the standard finite element shape functions and $u_{j}$ the associated weights. Because the contribution of $u^{S R}(x, y)$ in $\Omega_{l}, u_{j}$ do not correspond to the values of the unknown field $u(x, y)$ at the nodal positions $\left(x_{j}, y_{j}\right)$.

Remark.- We are assuming that the enrichment is performed by using standard finite elements, but in fact any kind of compact support approximation could be used. In order to represent interfaces involving weak discontinuities one could proceed within the standard finite element method by ensuring that the interface coincides with the elements edges. If the interface passes across the elements an enriched version of the finite element method should be considered (e.g. the extended finite element [19]). 
Now, to build-up the just defined approximation (19) we proceed as in the previous section by alternating a projection and an enrichment step until reaching convergence. The degrees of freedom related to the finite element contribution $u_{j}$ will be computed at the projection step because at this step we consider all the approximation functions known and we look for the associated weights. At the enrichment step a new functional couple of the separated representation will be calculated by assuming known all the previously computed functional couples as well as the just updated finite element enrichment. We are summarizing both steps.

\section{Projection stage}

At this stage we consider the test functions given by

$$
u^{*}(x, y)=\sum_{i=1}^{n} \alpha^{i *} F^{i}(x) \cdot G^{i}(y)+\sum_{j=1}^{N_{l}} \Psi_{j}(x, y) u_{j}^{*}
$$

which writes in the discrete form:

$$
u^{*}(x, y)=\sum_{i=1}^{n} \alpha^{i *} \mathbf{F}^{i T} \mathbf{M G}^{i T} \mathbf{N}+\mathbf{U}^{* T} \boldsymbol{\Psi}
$$

where $\boldsymbol{\Psi}$ is the vector that contains the finite element shape functions related to the set of nodes considered within the enrichment region $\Omega_{l}$, and $\mathbf{U}$ the vector that contains the nodal degrees of freedom.

Using the discrete form of function $u(x, y)$ :

$$
u(x, y)=\sum_{i=1}^{n} \alpha^{i} \mathbf{M}^{T} \mathbf{F}^{i} \mathbf{N}^{T} \mathbf{G}^{i}+\mathbf{\Psi}^{T} \mathbf{U}
$$

the weak formulation of Eq. (5) leads after numerical integration to the linear system

$$
\left(\boldsymbol{\alpha}^{* T} \mathbf{U}^{* T}\right)\left(\begin{array}{ll}
\mathbf{H} & \mathbf{B} \\
\mathbf{C} & \mathbf{Q}
\end{array}\right)\left(\begin{array}{c}
\boldsymbol{\alpha} \\
\mathbf{U}
\end{array}\right)=0
$$

In Eq. (23) matrix $\mathbf{H}$ is coming from the contribution of $u^{S R^{*}}$ and $u^{S R}$ and therefore it results the same that the one concerned in Eq. (10). On the other hand $\mathbf{Q}$ concerns the contribution coming from $u^{F E^{*}}$ and $u^{F E}$ that represents a classical finite element discretization in $\Omega_{l}$. The remaining terms $\mathbf{B}$ and $\mathbf{C}$ represent the crossing contributions.

In order to give an explicit form of the different matrices involved in Eq. (23), we introduce the following notation

$$
\left\{\begin{array}{l}
\mathbb{M}^{S R}=\int_{\Omega_{x}} \mathbf{M M}^{T} d \Omega_{x} \\
\mathbb{N}^{S R}=\int_{\Omega_{y}} \mathbf{N} \mathbf{N}^{T} d \Omega_{y} \\
\mathbb{K}^{S R}=\int_{\Omega_{x}} \mathbf{M} \mathcal{K} \mathbf{M}^{T} d \Omega_{x} \\
\mathbb{L}^{S R}=\int_{\Omega_{y}} \mathbf{N} \mathcal{L} \mathbf{N}^{T} d \Omega_{y}
\end{array}\right.
$$




$$
\begin{gathered}
\left\{\begin{array}{l}
\mathbb{K}^{F E}=\int_{\Omega_{l}} \mathbf{\Psi} \mathcal{K} \mathbf{\Psi}^{T} d \Omega \\
\mathbb{L}^{F E}=\int_{\Omega_{l}} \mathbf{\Psi} \mathcal{L} \mathbf{\Psi}^{T} d \Omega
\end{array}\right. \\
\left\{\begin{array}{l}
\mathbb{K}_{j}^{S R-F E}=\int_{\Omega_{l}} \mathbf{M N}^{T} \mathcal{K} \Psi_{j}^{T} d \Omega \\
\mathbb{L}_{j}^{S R-F E}=\int_{\Omega_{l}} \mathbf{M} \mathbf{N}^{T} \mathcal{L} \Psi_{j}^{T} d \Omega
\end{array}\right.
\end{gathered}
$$

and

$$
\left\{\begin{array}{l}
\mathbb{K}_{j}^{F E-S R}=\int_{\Omega_{l}} \Psi_{j} \mathcal{K} \mathbf{M} \mathbf{N}^{T} d \Omega \\
\mathbb{L}_{j}^{F E-S R}=\int_{\Omega_{l}} \Psi_{j} \mathbf{M} \mathcal{L} \mathbf{N}^{T} d \Omega
\end{array}\right.
$$

where, as mentioned in the previous section, these integrals take into account the specific character of each operator, as for example integration by parts or upwinding for stabilizing advective terms. These integrals, when they involves finite elements are performed using standard Gauss quadrature schemes. In the previous expressions $\Psi_{j}$ denotes the shape function related to node $j$, that is, the $j$-component of vector $\boldsymbol{\Psi}$.

Using these notations, the different matrices in Eq. (23) write:

$$
\begin{aligned}
H_{i j} & =\mathbf{F}^{i T} \mathbb{K}^{S R} \mathbf{F}^{j} \cdot \mathbf{G}^{i T} \mathbb{N}^{S R} \mathbf{G}^{j}+\mathbf{F}^{i T} \mathbb{M}^{S R} \mathbf{F}^{j} \cdot \mathbf{G}^{i T} \mathbb{L}^{S R} \mathbf{G}^{j} \\
Q_{i j} & =\left(\mathbb{K}^{F E}+\mathbb{L}^{F E}\right)_{i j} \\
B_{i j} & =\mathbf{F}^{i T}\left(\mathbb{K}_{j}^{S R-F E}+\mathbb{L}_{j}^{S R-F E}\right) \mathbf{G}^{i} \\
C_{i j} & =\mathbf{F}^{j T}\left(\mathbb{K}_{i}^{F E-S R}+\mathbb{L}_{i}^{F E-S R}\right) \mathbf{G}^{j}
\end{aligned}
$$

We can notice from the previous expressions that system (23) is nonsymmetric.

Eq. (23) must be solved again taking into account boundary and/or normality conditions.

\section{Enrichment stage}

At this stage we consider the unknown field given by

$$
u(x, y)=\sum_{i=1}^{n} \alpha^{i} F^{i}(x) \cdot G^{i}(y)+\sum_{j=1}^{N_{l}} \Psi_{j}(x, y) \cdot u_{j}+R(x) \cdot S(y)
$$

where the first two sums in the right member are assumed known (computed at the projection stage).

The test function reads:

$$
u^{*}(x, y)=R^{*}(x) \cdot S(y)+R(x) \cdot S^{*}(y)
$$


whose discrete form writes

$$
u^{*}(x, y)=\left[\begin{array}{ll}
\mathbf{R}^{* T} & \mathbf{S}^{* T}
\end{array}\right]\left(\begin{array}{c}
\mathbf{M}\left(\mathbf{S}^{T} \mathbf{N}\right) \\
\left(\mathbf{R}^{T} \mathbf{M}\right) \mathbf{N}
\end{array}\right)
$$

The weak form of Eq. (5) becomes, in the framework of a fixed point strategy, in:

- For a given $S(y)$ (that implies $u^{*}(x, y)=R^{*}(x) \cdot S(y)$ )

$$
\begin{aligned}
& \left(\mathbb{K}^{S R} \mathbf{S}^{T} \mathbb{N}^{S R} \mathbf{S}+\mathbb{M}^{S R} \mathbf{S}^{T} \mathbb{L}^{S R} \mathbf{S}\right) \mathbf{R}= \\
& =-\sum_{i=1}^{n} \alpha^{i}\left(\mathbb{K}^{S R} \mathbf{F}^{i} \mathbf{S}^{T} \mathbb{N}^{S R} \mathbf{G}^{i}+\mathbb{M}^{S R} \mathbf{F}^{i} \mathbf{S}^{T} \mathbb{L}^{S R} \mathbf{G}^{i}\right)-\mathcal{H}^{R-F E}
\end{aligned}
$$

where the finite element enrichment contribution $\mathcal{H}^{R-F E}$ writes:

$$
\mathcal{H}^{R-F E}=\sum_{j=1}^{j=N_{l}}\left(\left(\mathbb{K}_{j}^{S R-F E}+\mathbb{L}_{j}^{S R-F E}\right) \mathbf{S}\right) u_{j}
$$

- For a given $R(x)$ (that implies $u^{*}(x, y)=R(x) \cdot S^{*}(y)$ )

$$
\begin{aligned}
& \left(\mathbf{R}^{T} \mathbb{K}^{S R} \mathbf{R} \mathbb{N}^{S R}+\mathbf{R}^{T} \mathbb{M}^{S R} \mathbf{R} \mathbb{L}^{S R}\right) \mathbf{S}= \\
& =-\sum_{i=1}^{n} \alpha^{i}\left(\mathbf{R}^{T} \mathbb{K}^{S R} \mathbf{F}^{i} \mathbb{N}^{S R} \mathbf{G}^{i}+\mathbf{R}^{T} \mathbb{M}^{S R} \mathbf{F}^{i} \mathbb{L}^{S R} \mathbf{G}^{i}\right)-\mathcal{H}^{S-F E}
\end{aligned}
$$

where the finite element enrichment contribution $\mathcal{H}^{S-F E}$ writes:

$$
\mathcal{H}^{S-F E}=\sum_{j=1}^{j=N_{l}}\left(\left(\mathbb{K}_{j}^{S R-F E}+\mathbb{L}_{j}^{S R-F E}\right)^{T} \mathbf{R}\right) u_{j}
$$

It must be noticed that the number of the degrees of freedom involved in such non linear solution is the sum of the degrees of freedom involved in each one-dimensional discretization, instead the product of them that results in mesh based discretization strategies.

Finally, after convergence, the new approximation functions $\mathbf{F}^{n+1}$ and $\mathbf{G}^{n+1}$ are obtained by normalizing $\mathbf{R}$ and $\mathbf{S}$ :

$$
\left\{\begin{array}{l}
\mathbf{F}^{n+1}=\mathbf{R}\left(\mathbf{R}^{T} \mathbb{M} \mathbf{R}\right)^{-\frac{1}{2}} \\
\mathbf{G}^{n+1}=\mathbf{S}\left(\mathbf{S}^{T} \mathbb{N} \mathbf{S}\right)^{-\frac{1}{2}}
\end{array}\right.
$$

The projection and enrichment stages must continue until reaching convergence. Two simple possibilities exist: (i) evaluating the norm of $R(x)$ and $S(y)$ and use these norms as stopping criterion (this criterion does not guarantee the convergence), or (ii) evaluate the norm of the strong formulation residual that is more expensive but it guarantees the convergence. In what follows we are using the second stopping criterion. 


\section{A first numerical example involving localiza- tion}

To illustrate the capabilities of the just described strategy we are solving a simple Poisson equation

$$
-\Delta u=s(x, y), \quad(x, y) \in \Omega=\left(-\frac{\pi}{2}, \frac{\pi}{2}\right) \times\left(-\frac{\pi}{2}, \frac{\pi}{2}\right)
$$

with homogeneous boundary conditions, i.e.

$$
u(\mathbf{x} \in \partial \Omega)=0
$$

The source term $s(x, y)$ considered in the numerical experiment described in this section was:

$$
s(x, y)=1+\frac{-4 f\left(s_{1}+s_{2} s_{3}\right)}{s_{4}}
$$

with

$$
\left\{\begin{array}{l}
s_{1}=-1+2 d^{2} f+f x^{2}+f y^{2}+2 d f(x+y) \\
s_{2}=e^{4 d f(x+y)} \\
s_{3}=\left(-1+2 d^{2} f-2 d f(x+y)+f\left(x^{2}+y^{2}\right)\right) \\
s_{4}=e^{f\left(2 d^{2}+x^{2}+y^{2}+2 d(x+y)\right)}
\end{array}\right.
$$

where coefficient $d$ controls the location of the localized behavior whereas coefficient $f$ controls the support of such localized behavior. In the numerical experiment that follows we considered $f=40$ and $d=1.4$. The above source term admits a separated representation involving 5 functional products.

The particular form of this source term leads to a localized behavior of the solution in the neighborhood of the upper-right and lower-left corners. This behavior allows to analyze the improvement that an eventual finite element based enrichment of the separated representation could bring.

First, the problem is solved using the standard finite element method. Then, it is solved again using a fully separated representation. Obviously, the existence of a localized behavior requires a fine enough resolution in both directions (one-dimensional meshes fine enough to capture the localized behavior) and also numerous functional products for approximating accurately the solution behavior.

Finally, the use of a separated representation combined with a finite element enrichment in the regions where the solution exhibits high gradients allows to define a technique sharing the benefits of both the finite elements and the separated representations.

Figure 1 depicts the mesh considered for solving problem (40)-(42) by using the finite element method. The associated solution is depicted in figure 2 .

Now, we are solving the same problem by using a coarse fully separated representation. The solution was computed by applying the technique described 


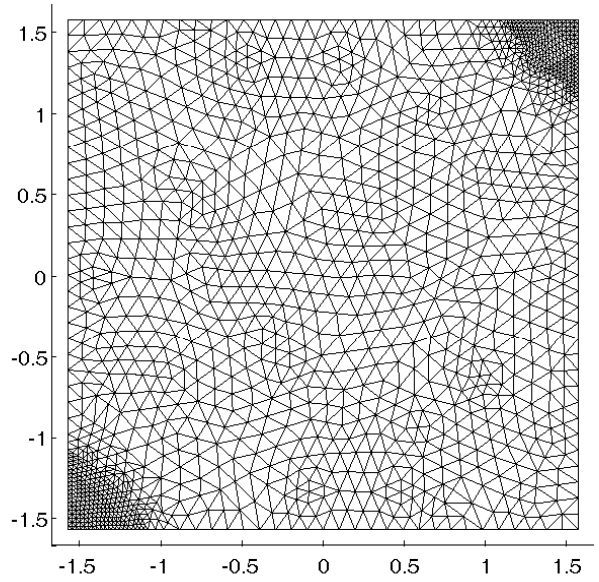

Figure 1: Finite element mesh.

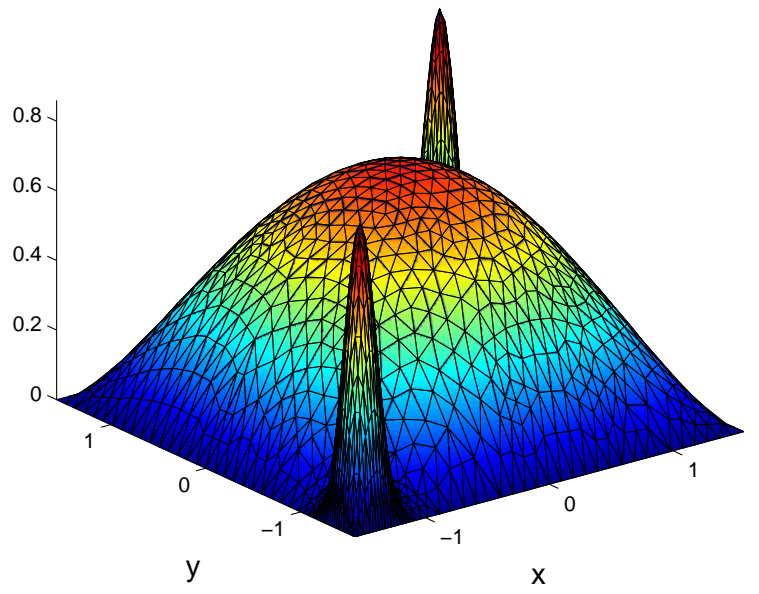

Figure 2: Finite element solution. 
in section 2. We consider a very coarse separated representation, involving 7 nodes on each direction. As expected, the computed solution depicted in Fig. 3 does not account for the presence of the localized behavior. Figs. 4 depicts the one-dimensional functions associated with the coordinates $x, F^{i}(x)$ (on the left) and $y, G^{i}(y)$ (on the right), involved in the separated representation.

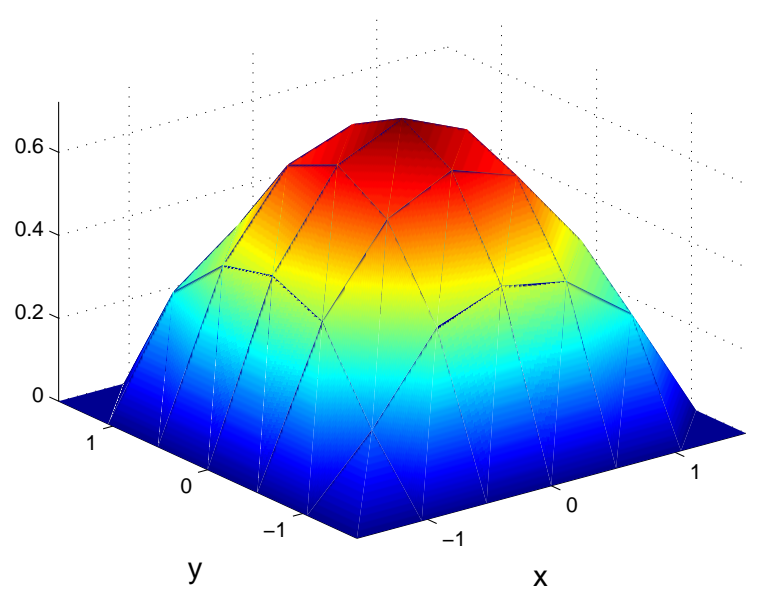

Figure 3: Solution computed using a coarse separated representation.
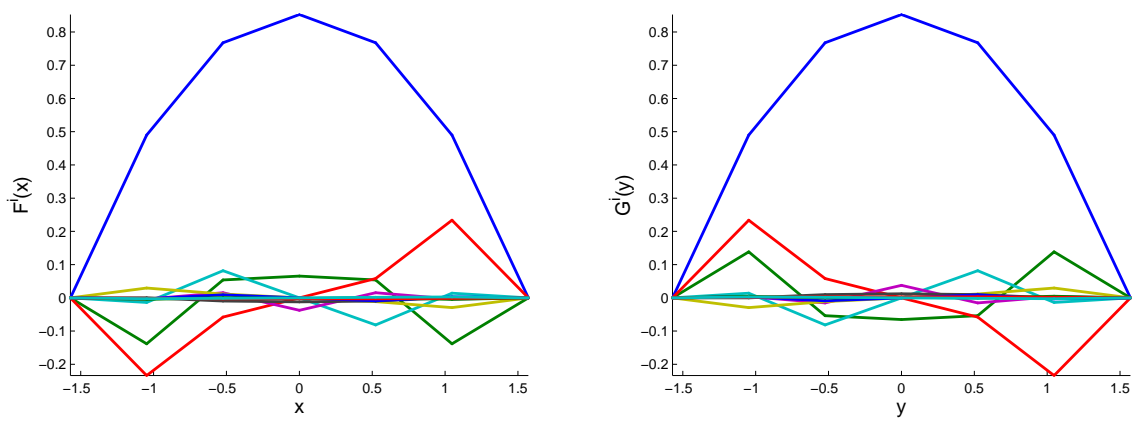

Figure 4: One-dimensional functions involved in the separated representation: $F^{i}(x)$ (left) and $G^{i}(y)$ (right).

In this case, the regions in which the solution exhibits localization remain invisible to the separated approximation. Obviously, finer approximations of the one-dimensional approximation involved in the separated representation could finally capture the solution localized behavior. Instead of proceeding by refining the separated representation, our goal is capturing the localized behavior by 
superposing a finite element approximation in the regions exhibiting localization.

Figure 5 depicts the finite element meshes considered in the regions where the solution localization is expected.

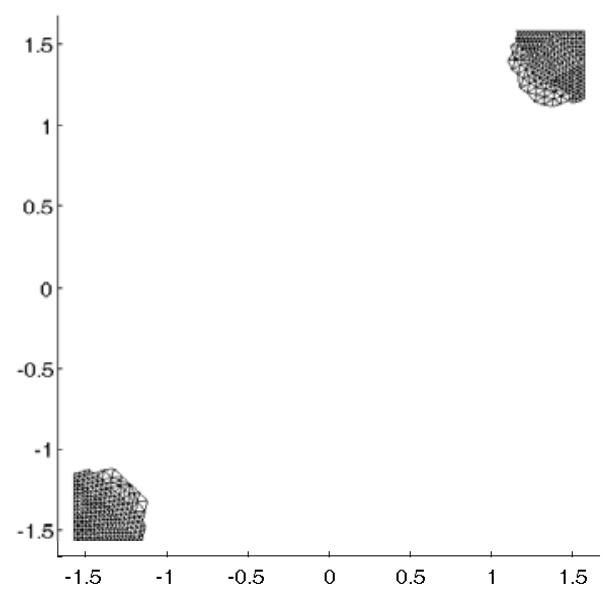

Figure 5: Finite element meshes on the regions that exhibit localization.

Now, we are using the finite element based enrichment of the separated representation, that is the mixed approximation that involves the separated form plus the contribution of the finite element approximation that is superposed to the former one in the regions depicted in Fig. 5. In this case the computed solution is shown in Fig. 6.

Obviously, as the one-dimensional meshes considered for defining the interpolations of functions $F^{i}(x)$ and $G^{i}(y)$ are too coarse to detect the presence of the localized behavior, the contribution of the separated representation to the whole solution depicted in Fig. 6 coincides to the one computed previously by using a fully separated representation (Fig. 3) and in consequence functions $F^{i}(x)$ and $G^{i}(y)$ involved in the enriched separated representation also coincide to the ones depicted in Fig. 4. Obviously, finer separated representations could represent a part of the localized behavior.

Obviously, the finite element enrichment captures the localized behavior as Fig. 7 depicts. This figure only represents the finite element contribution. We can notice that by construction, the finite element approximation vanishes on the boundary of the finite element meshes.

The coarse separated representation without the finite element representation involves a $L^{2}$ error of 0.23 (with respect to the finite element solution previously depicted). Obviously, the introduction of more terms in the finite sums decomposition cannot improve significantly this error because the onedimensional meshes cannot capture the localized behaviors as we commented previously. However, as soon as the finite element enrichment applies the error 


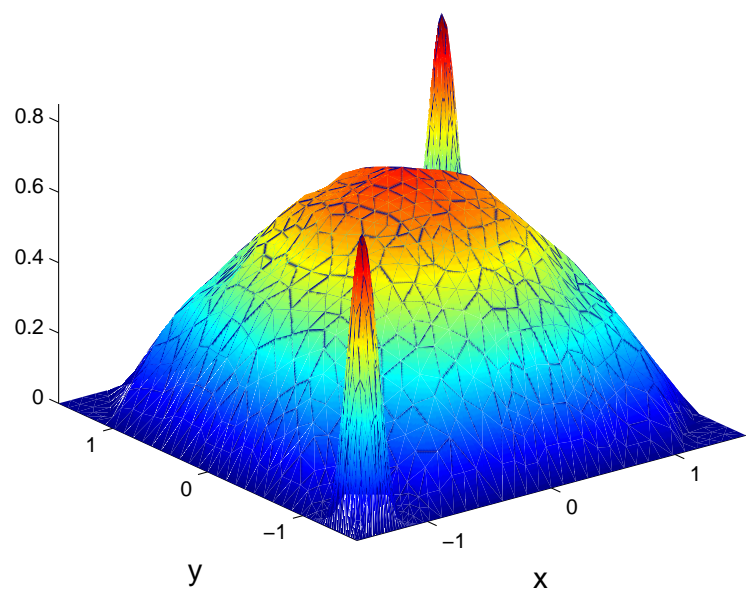

Figure 6: Computed solution using a finite element enriched separated representation.

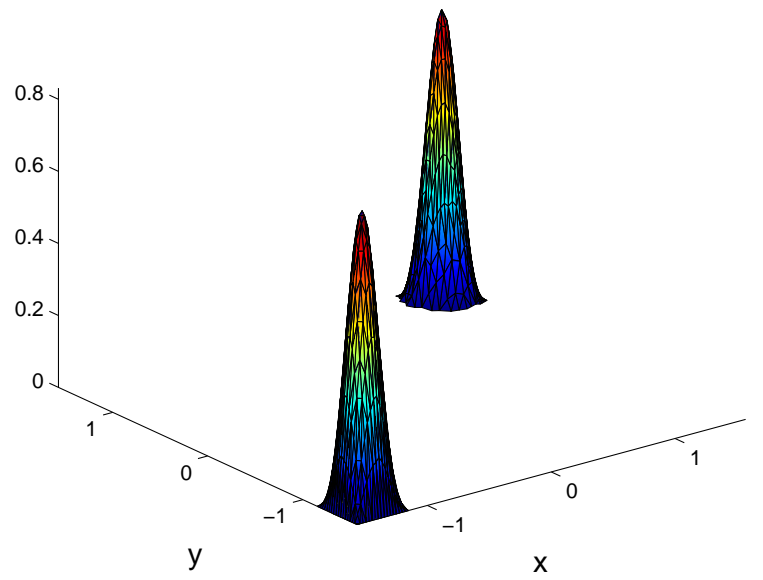

Figure 7: Finite element approximation contribution. 
is drastically reduced to 0.09. For further error reductions finer finite element meshes should be considered. The use of finer separated representations could be also a strategy, but the effect of finite element remeshing is more efficient, because finite element interpolations are more adapted to capture localized behaviors.

\section{$5 \quad$ A strategy for coupling subdomains}

The enrichment procedure presented in this paper opens new possibilities for extending the applicability of separated representations. One of these potential applications lies in the coupling of subdomains, each one involving a separated approximation of the model variable. To illustrate the coupling procedure just described we are considering the solution of the model

$$
-\Delta u=2\left(y-y^{2}\right)+2\left(1-x^{2}\right), \quad(x, y) \in \Omega=(-1,1) \times(0,1)
$$

with homogeneous boundary conditions, i.e.

$$
u(\mathbf{x} \in \partial \Omega)=0
$$

The exact solution writes

$$
u^{e x}(x, y)=\left(1-x^{2}\right)\left(y-y^{2}\right)
$$

The domain $\Omega=(-1,1) \times(0,1)$ can be decomposed in two subdomains $\Omega_{1}=(-1,0) \times(0,1)$ and $\Omega_{2}=(0.1) \times(0,1)$ such that $\Omega=\Omega_{1} \cup \Omega_{2}$ and $\Gamma=\bar{\Omega}_{1} \cap \bar{\Omega}_{2}$ (being $\Gamma$ the interface between both subdomains). It is easy to understand that the solution of such one model is quite delicate because we should build-up a separated approximation of the solution in both subdomains assuring the appropriate transfer conditions across the interface $\Gamma$. Obviously, in the scenario just described the best alternative is the solution of the problem directly in the whole domain $\Omega$ because as it is rectangular, its decomposition in two subdomains is not needed. However, other kind of domains, e.g. L or T shaped domains, need appropriate subdomain decompositions.

An alternative lies in the construction of a separated representation in both subdomains assuming that both separated representations vanish on the interface $\Gamma$. Now, a finite element based enrichment is introduced in a strip $\Omega_{l}$ of width $2 H$ covering the interface, $\Omega_{l}=(-H,+H) \times(0,1)$. As described in section 3 , the nodal degrees of freedom related to nodes located on $\partial \Omega_{l}$ are enforced to vanish, to ensure the compatibility (continuity) of the approximations across the enrichment domain boundary as well as for accounting the boundary conditions prescribed on $\partial \Omega$. Thus, the finite element model defined in $\Omega_{l}$ ensures the compatibility of the solutions computed in both subdomains in a finite element sense.

To perform the coupling of the model described above, we consider a strip covering the interface, $\Omega_{l}=(-H,+H) \times(0,1)$, with $H=0.25$, that is meshed 


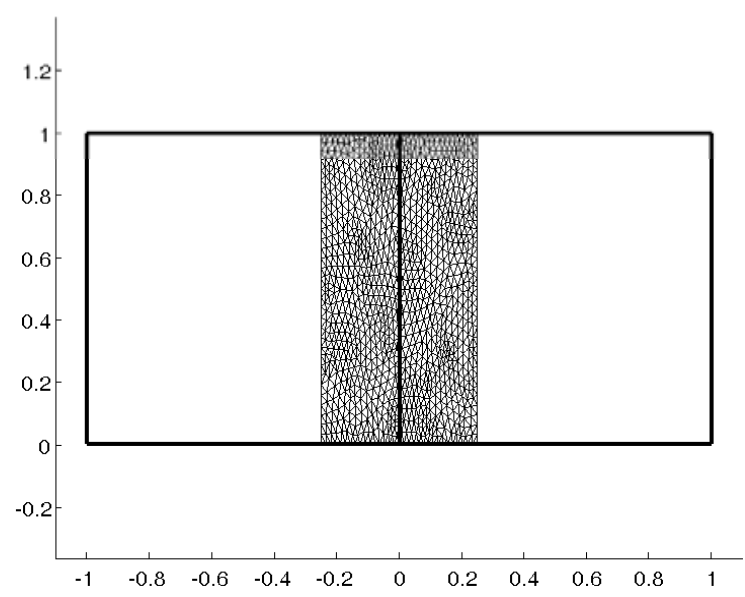

Figure 8: Domain decomposition.

using linear triangular finite elements. Fig. 8 shows the domain decomposition as well as the mesh of $\Omega_{l}$.

The exact solution is depicted in Fig. 9. Now, the solution in the whole domain is computed by applying the strategy just described. Figure 10 depicts the separated representation solution in $\Omega_{1}$ (left) and $\Omega_{2}$ (right) as well as the finite element enrichment in $\Omega_{l}$ (center). We can see that the separated representation contribution in both domains vanish by construction on $\partial \Omega_{1}$ and $\partial \Omega_{2}$. Thus, the separated representation contributions do not introduce any continuity issue across the interface. The accomplishment of the transmission conditions (in our case the field continuity) is ensured by the finite element contribution that does not compromise the required continuity.

Fig. 11 represents the functions $F^{i}(x)$ and $G^{i}(y)$ related to the separated representation contribution in domain $\Omega_{1}$. The ones concerning the domain $\Omega_{2}$ can be obtained from these ones: functions of the $x$-coordinate are derived by symmetry considerations, and the ones concerning the $y$-coordinate are exactly the same that the corresponding ones in domain $\Omega_{1}$.

By combining the separated representation and the finite element contribution we can define the solution in the whole domain. The combined solution is depicted in Fig. 12. Fig. 13 depicts the error in the resulting solution for different number of terms in the finite sums decomposition of the separated representations (the finite element mesh remains unchanged). The solutions obtained by using a single term in the separated representations define the so-called Hartree approximations. Finally, Fig. 14 depicts the evolution of the $L^{2}$-norm of the error as a function of the number of terms involved in the separated representation. As expected, the error stabilizes when the number of terms increases, and further reduction needs both finer separated representations (involving finer 


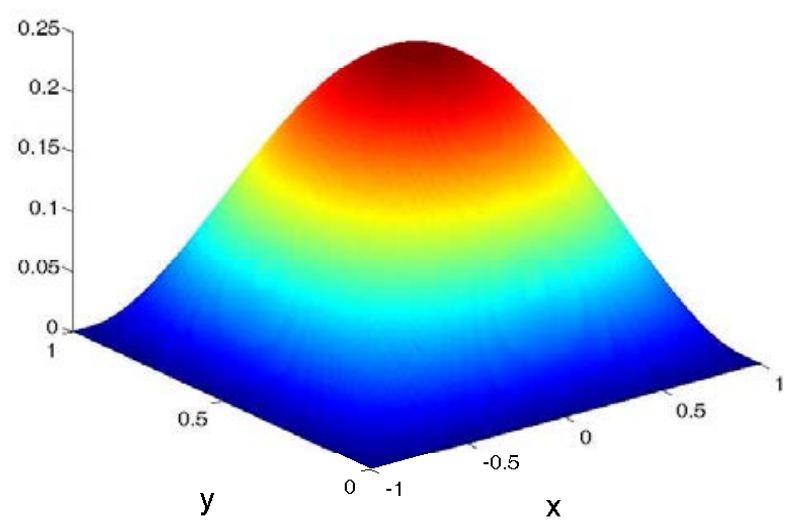

Figure 9: Reference solution.
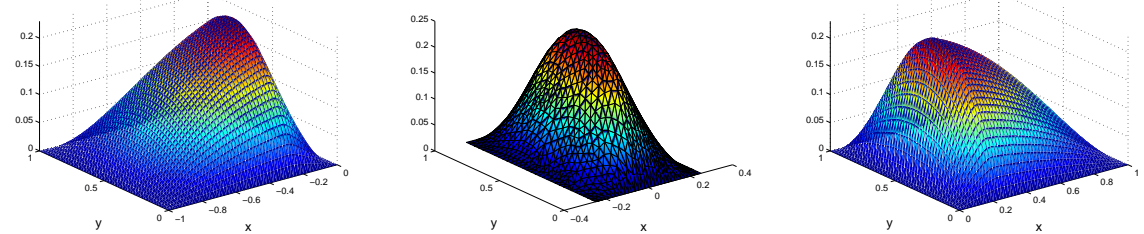

Figure 10: Contributions to the whole solution: separated representation in $\Omega_{1}$ (left); finite element contribution (center) and separated representation in $\Omega_{2}$ (right).
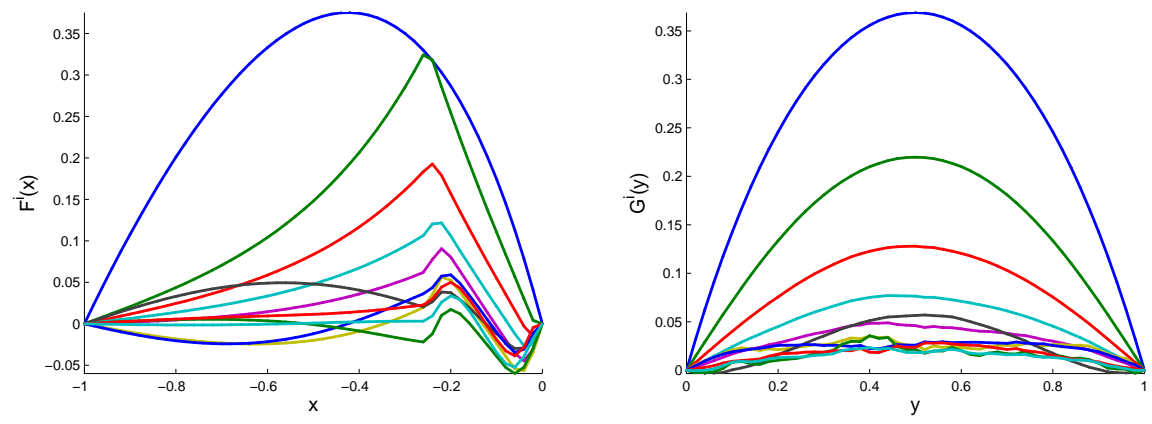

Figure 11: Functions $F^{i}(x)$ (left) and $G^{i}(y)$ involved by the separated representation in domain $\Omega_{1}$. 
one-dimensional meshes) and finer finite element meshes.

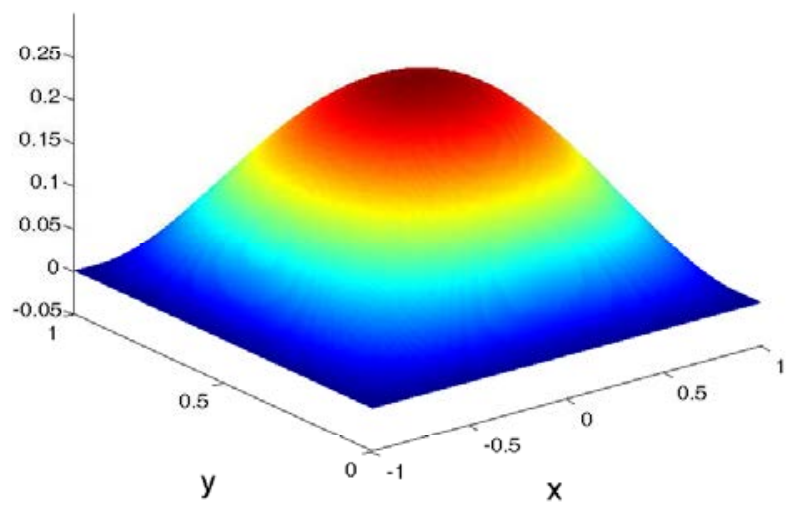

Figure 12: Computed solution in the whole domain resulting of the combination of contributions to the solution depicted in Fig. 10.
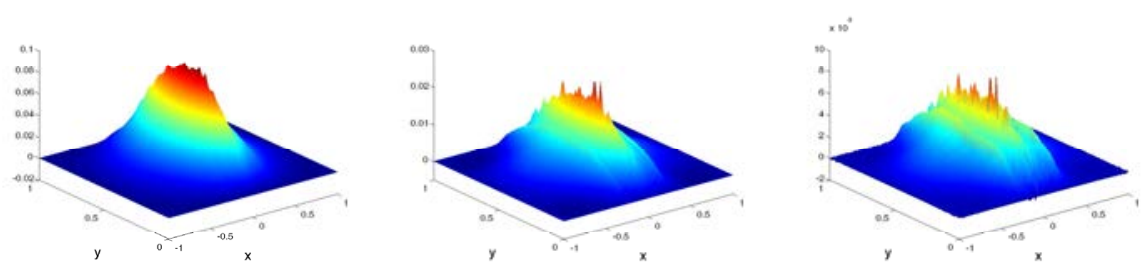

Figure 13: Error when the separated representations involve 1 (left), 3 (center) and 10 terms (right).

\section{Conclusions and perspectives}

The proposed strategy can be used for enriching separated representations in order to take into account localized behavior of the solution. A numerical example proved the ability of defining reduced approximation bases able to capture localized behavior.

This strategy can be also used for treating models involving strong or weak discontinuities. In this case the separated representation should be coupled with an appropriate finite element enrichment able to describe the involved discontinuities. There is an extensive literature concerning the use of finite elements for this purpose. 


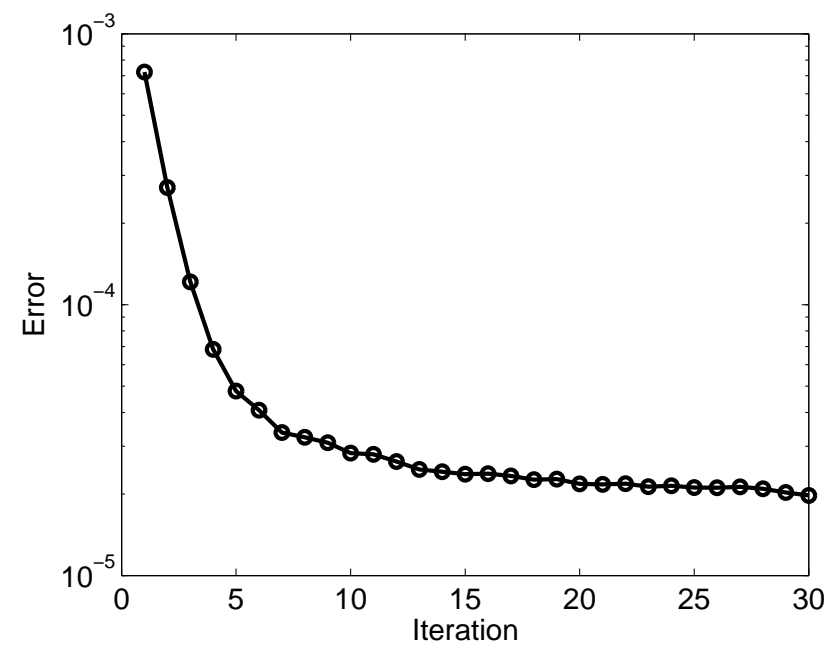

Figure 14: Error versus number of terms involved in the separated representations.

There are many other potential applications. One could use this technique to define bridges between finite element models and other making use of proper generalized decompositions, or to make compatible subdomains making use of proper generalized decompositions involving different functional products or different one dimensional meshes on the common interface. It could be also applied for enforcing general boundary conditions by introducing a finite element description in a strip along the domain boundary, or to define proper generalized decompositions in geometrically complex domains. The analysis of these and other possibilities will be addressed in future works.

\section{Acknowledgements}

This work has been partially supported by the Spanish Ministry of Science and Innovation, through grant number CICYT-DPI2008-00918. This support is gratefully acknowledged.

\section{References}

[1] Ammar A., Mokdad B., Chinesta F., Keunings R., A new family of solvers for some clases of multidimensional partial differential equations encountered in kinetic theory modeling of complex fluids, Journal of NonNewtonian Fluid Mechanics, 139, 2006, 153-176. 
[2] Ammar A., Mokdad B., Chinesta F., Keunings R., A new family of solvers for some classes of multidimensional partial differential equations encountered in kinetic theory modeling of complex fluids. Part II: Transient simulation using space-time separated representation. Journal of Non-Newtonian Fluid Mechanics, 144, 2007, 98-121.

[3] Ammar A., Normandin M., Daim F., Gonzalez D., Cueto E., Chinesta F., Non-incremental strategies based on separated representations: Applications in computational rheology. Communications in Mathematical Sciences, In press.

[4] Ammar A., Chinesta F., Falco A., A Kronecker product based iterative solvers for linear systems, Numerical Linear Algebra with Applications, Submitted

[5] Ammar A., Normandin M., Daim F., Gonzalez D., Cueto E., Chinesta F., Non-incremental strategies based on separated representations: Applications in computational rheology, Communications in Mathematical Sciences, Submitted.

[6] Bungartz H.J., Griebel M., Sparse grids, Acta Numerica, 13, 2004, 1-123.

[7] Cancès E., Defranceschi M., Kutzelnigg W., Le Bris C., Maday Y., Computational Quantum Chemistry: a primer, Handbook of Numerical Analysis, Vol. X, Elsevier, 2003, pp. 3-270.

[8] F. Chinesta, A. Ammar, A. Falco et M. Laso., On the reduction of stochastic kinetic theory models of complex fluids. Modeling and Simulation in Materials Science and Engineering, 15, 2007, pp. 639-652.

[9] Chinesta F., Ammar A., Joyot P., The nanometric and micrometric scales of the structure and mechanics of materials revisited: An introduction to the challenges of fully deterministic numerical descriptions. International Journal for Multiscale Computational Engineering, 6/3, 2008, 191-213.

[10] Chinesta F., Ammar A., Lemarchand F., Beauchene P., Boust F., Alleviating mesh constraints: Model reduction, parallel time integration and high resolution homogenization. Computer Methods in Applied Mechanics and Engineering, 197/5, 2008, 400-413.

[11] J. Fish and R. Guttal. The s-version of the finite element method for laminated composites. International Journal for Numerical Methods in Engineering, 39, 3641-3662, 1996.

[12] Gonzalez D., Ammar A., Chinesta F., Cueto E., Recent advances in the use of separated representations, International Journal for Numerical Methods in Engineering, Submitted.

[13] Ladeveze P., Non linear computational structural mechanics, Springer, N.Y., 1999. 
[14] Ladeveze P., Passieux J.Ch., Neron D., The LATIN multiscale computational method and the proper orthogonal decomposition, Computer Methods in Applied Mechanics and Engineering, In press

[15] Nouy A., Ladevze P., Multiscale computational strategy with time and space homogenization: A radial-type approximation technique for solving microproblems, International Journal of Multiscale Computational Engineering, $\mathbf{1 7 0 / 2}, 2004$.

[16] Nouy A., Recent developments in spectral stochastic methods for the solution of stochastic partial differential equations, Archives of Comput. Meth. In Engrg., In press.

[17] E. Rank and R. Krause, A multiscale finite element method, Computers and Structures, 64 (1-4), 139-144, 1997.

[18] Sreenath S.N., Cho K-H, Wellstead P., Modelling the dynamics of signalling pathways, Essays in biochemistry, 45, 2008, 1-28.

[19] Sukumar N., Chopp D., Moes N., Belytschko T., Modeling holes and inclusions by level sets in the extended finite element method, Comput. Methods Appl. Mech. Engrg. 190, 2001, 6183-6200. 